Pesq. Vet. Bras. 37(2):156-159, fevereiro 2017

DOI: $10.1590 / \mathrm{S} 0100-736 \mathrm{X} 2017000200010$

\title{
Influência de diferentes protocolos de contenção nos valores do eletrocardiograma de macacos-da-noite (Aotus azarae infulatus) ${ }^{1}$
}

\author{
Rosa H.F. Chaves ${ }^{2 *}$, Nazaré F. Souza ${ }^{3}$, José A.P.C. Muniz ${ }^{4}$, Aline A. Imbeloni ${ }^{4}$, \\ Ana C.S. Neves ${ }^{3}$ e Renan K.C.Teixeira ${ }^{2}$
}

\begin{abstract}
Chaves R.H.F., Souza N.F., Muniz J.A.P.C., Imbeloni A.A., Neves A.C.S. \& Teixeira R.K.C. 2017. [Influence of different restraint protocols for the electrocardiogram values of feline night monkeys (Aotus azarae infulatus).] Influência de diferentes protocolos de contenção nos valores do eletrocardiograma de macacos-da-noite (Aotus azarae infulatus). Pesquisa Veterinária Brasileira 37(2):156-159. Laboratório de Cirurgia Experimental, Centro de Ciências Biológicas e da Saúde, Universidade do Estado do Pará, Travessa Perebebuí 2623, Belém, PA 66087-670, Brazil. E-mail: rosinhahelena@gmail.com

Feline night monkeys are very susceptible to stress and therefore the physical or chemical restraint should be carefully evaluated before any procedure. Anesthetic protocols can alter some physiological parameters, and electrocardiogram (ECG) is a commonly exam used for evaluation of the rhythm and heart rate. The objective of this study was to evaluate the influence of four different protocols of restraining on ECG in Aotus azarae infulatus, in order to identify the best method of restraining in these primates. For that, we used 10 adult feline night monkeys, males, submitted to restraining protocols with the association tiletamine and zolazepam - (TZ), isoflurane (ISO), association ketamine and midazolam (CET) and physical restraint (CF). No differences were observed in the parameters wave and ECG complexes obtained in all groups tested; however, during the evaluation of the trace the animals of group CF showed a greater amount of change. It was concluded that the restraint protocols used did not change the values of the ECG, and it was not possible to consider the CF group as control due to more caused alterations than all other tested protocols.
\end{abstract}

INDEX TERMS: Restraint protocols, electrocardiogram, feline night monkeys, Aotus azarae infulatus, anesthesia.

RESUMO.- Macacos-da-noite são muito susceptíveis ao estresse e por isso a contenção química ou física deve ser cuidadosamente avaliada antes de qualquer procedimento. Protocolos anestésicos podem alterar alguns parâmetros fisiológicos, sendo o eletrocardiograma (ECG) um exame muito utilizado para avaliação do ritmo e da frequência cardíaca. 0 objetivo deste estudo foi avaliar a influência de quatro diferentes protocolos de contenção sobre o ECG rea-

\footnotetext{
${ }^{1}$ Recebido em 20 de maio de 2016.

Aceito para publicação em 2 de dezembro de 2016

${ }^{2}$ Laboratório de Cirurgia Experimental, Centro de Ciências Biológicas e da Saúde, Universidade do Estado do Pará (UEPA), Travessa Perebebuí 2623, Belém, PA 66087-670, Brasil. *Autor para correspondência: rosinhahelena@gmail.com

${ }^{3}$ Instituto de Saúde e Produção Animal, Universidade Federal Rural da Amazônia (UFRA), Av. Presidente Tancredo Neves 2501, Belém, PA 66077901, Brasil.

${ }^{4}$ Centro Nacional de Primatas (CENP), Rodovia BR-316 Km 7 s/n, Ananindeua, PA 67030-000, Brasil.
}

lizado em Aotus azarae infulatus. Para isso foram utilizados 10 animais, machos, adultos, submetidos à contenção com a associação tiletamina/zolazepam (TZ), isoflurano (ISO), associação cetamina e midazolam (CET) e contenção física (CF). Não foram observadas diferenças nos parâmetros de ondas e complexos obtidos no ECG em todos os grupos testados, no entanto, durante a avaliação do traçado os animais do grupo $\mathrm{CF}$ apresentaram uma quantidade maior de alterações. Concluiu-se que os protocolos de contenção utilizados não alteraram os valores do ECG e que não foi possível considerar o grupo CF como controle devido causar mais alterações do que todos os protocolos testados.

TERMOS DE INDEXAÇÃO: Contenção, eletrocardiograma, macacos-da-noite, Aotus azarae infulatus, anestesia.

\section{INTRODUÇÃO}

O Brasil abriga a maior diversidade de primatas do planeta, com cerca de 135 táxons reconhecidos para o país (ICMBIO 
2011), sendo que destes, 70\% estão localizados na Amazônia, o que confere a essa região um verdadeiro atrativo para estudos de biodiversidade (Del-Claro \& Prezoto 2003). Macacos-da-noite (Aotus azarae infulatus) são pequenos primatas neotropicais com hábitos noturnos únicos e são extensivamente usados como modelos animais para pesquisa biomédica (Menezes et al. 2010). São extremamente susceptíveis ao estresse quando manipulados e, por isso, a utilização de fármacos anestésicos e métodos de contenção física devem ser cuidadosamente avaliados antes de qualquer procedimento (Monteiro 2004).

Diversas pesquisas têm sido realizadas com o objetivo de descobrir o tranquilizante e o anestésico ideais, dotados de menor poder de toxicidade e que possam ser usados em indivíduos de idade avançada, debilitados, facilmente estressáveis e, ainda, que sejam de baixo custo e de fácil aquisição (Silva \& Ferreira 1994).

0 eletrocardiograma (ECG) é um método gráfico obtido quando os potenciais de um campo elétrico com origem no coração são registrados à superfície do organismo. É o exame auxiliar mais utilizado no diagnóstico de doenças cardíacas por ser um exame não invasivo e barato, além de fornecer informações valiosas acerca da orientação anatômica do coração, tamanho relativo das diversas câmaras cardíacas, frequência cardíaca (FC) e uma variedade de alterações do ritmo e condução elétrica (Chaves \& Moreira 2001).

0 objetivo desse estudo foi avaliar a influência de quatro diferentes protocolos de contenção sobre o eletrocardiograma realizado em primatas não-humanos neotropicais da espécie Aotus azarae infulatus (macaco-da-noite), com o intuito de identificar qual o melhor método de contenção para a realização de procedimentos biomédicos nestes animais.

\section{MATERIAL E MÉTODOS}

O projeto foi aprovado pela Comissão de Ética no Uso de Animais da Unesp-Botucatu sob protocolo no.215/2012-CEUA, e pelo Ministério do Meio Ambiente - Sistema de Autorização e Informação em Biodiversidade (Sisbio) sob protocolo de cadastramento de número 37034. Foram utilizados 10 (dez) Macacos-da-noite (Aotus azarae infulatus), adultos, machos, com peso médio de 1 $\mathrm{kg}$, clinicamente hígidos, pertencentes ao Centro Nacional de Primatas (Cenp - Ananindeua, Pará, Brasil, latitude 1³8'26” e longitude $48^{\circ} 38^{\prime} 22^{\prime \prime}$ ). Foram realizados a avaliação clínica e exames de hemograma e bioquímica sérica (alanina aminotransferase - ALT, aspartato aminotransferase - AST, fosfatase alcalina, ureia, creatinina e glicemia) no período de seleção dos animais, que ocorreu 15 dias antes do início dos procedimentos. Os resultados destes exames foram comparados aos valores de referência para esta espécie, sendo selecionados para o experimento apenas aqueles considerados hígidos. A necessidade de utilizar os mesmos animais para os quatro protocolos de contenção, deve-se à necessidade de observar as possíveis alterações eletrocardiográficas apresentadas por um mesmo organismo frente aos procedimentos realizados. Foi realizada pesagem prévia para que fosse calculada a quantidade exata de anestésico a ser administrada, em seguida os animais foram submetidos aos protocolos com intervalo de uma semana entre eles.

TZ: neste grupo foi utilizada a associação tiletamina e zolazepam, na dose de 5,5 mg. $\mathrm{kg}^{-1}$ pela via intramuscular;

ISO: neste grupo foi utilizado o anestésico isoflurano, fornecido através de máscara facial, liberando-se o fluxo do anestésico associado ao oxigênio;

CET: neste grupo foi utilizada associação de $15 \mathrm{mg} . \mathrm{kg}^{-1}$ de cloridrato de cetamina e $1 \mathrm{mg} \cdot \mathrm{kg}^{-1}$ de midazolam, associados na mesma seringa pela via intramuscular;

CF: neste grupo os animais foram contidos com o auxílio de luvas de raspa de couro, em posição supina, de forma semelhante ao descrito por Monteiro et al. (2006).

Após o exame eletrocardiográfico, os animais submetidos à anestesia foram mantidos em gaiola até o seu retorno anestésico completo. Para a realização do eletrocardiograma, o animal foi contido em posição supina, posicionando-se os eletrodos na região do cotovelo direito, (1) e esquerdo (1) e nas pernas, na região do joelho direito (1) e esquerdo (1). Para determinar as precordiais os eletrodos foram posicionados no $5^{\circ}$ espaço intercostal (EIC) direito, próximo ao esterno (V1), no $6^{\circ}$ EIC esquerdo, próximo à região do esterno (V2), no $6^{\circ} \mathrm{EIC}$, linha axilar média, (V3), no $6^{\circ}$ EIC, linha axilar posterior (V4). Neste estudo não foram usados os 6 eletrodos das derivações precordiais, devido ao tamanho reduzido da caixa torácica dos animais. Para a realização do exame, utilizou-se o eletrocardiógrafo (CardioCare 2000 Bionet, Seoul, Coréia do Sul, Registro ANVISA no: 800702100004), nas velocidades 25 e $50 \mathrm{~mm} / \mathrm{s}$.

Para mensuração dos parâmetros do ECG foram levados em consideração apenas ondas e complexos corretos, pois estes permitem a determinação do tamanho de cada segmento. Ao final, o traçado foi avaliado de forma geral, no intuito de observar se houve alguma alteração do padrão, sendo neste item pontuadas as alterações encontradas.

Todos os dados foram avaliados quanto à normalidade pelo teste de Shapiro-Wilk e homogeneidade por Levene. Para avaliação das ondas e complexos, os dados foram submetidos à análise de variância (ANOVA 1 critério) seguida pelo Teste de Tukey, além dos testes de Wilcoxon e Correlação Linear de Pearson. 0 teste Qui-quadrado foi utilizado para analisar as frequências de ocorrência das alterações. Foram considerados significativos valores de $p$ bilateral igual ou inferior a $5 \%(p \leq 0,05)$.

\section{RESULTADOS}

Durante a avaliação dos animais, observou-se que os exames de hemograma e bioquímica sérica apresentaram-se normais quando comparados ao padrão da espécie, e por isso todos os animais pré-selecionados foram incluídos no estudo.

0 Quadro 1 resume os parâmetros obtidos a partir do traçado eletrocardiográfico.

Não foram encontradas diferenças estatisticamente significativas nos valores de FC em todos os grupos testados. Com relação ao eixo cardíaco, não houve diferença entre o eixo dos grupos. Foi verificado ritmo sinusal com marca-passo migratório em um animal do grupo CF (2,5\%), enquanto que todos os outros apresentaram ritmo sinusal normal $(97,5 \%)$.

Não foram observadas diferenças entre os valores de onda P (comprimento e altura), intervalo P-R, complexo QRS (comprimento e altura), segmento ST, e intervalo Q-T e polaridade de onda T em todos os grupos testados.

Os valores obtidos a partir das derivações precordiais estão dispostos no Quadro 2.

Não houve diferença estatística em V1 nos valores das ondas $\mathrm{R}$ e $\mathrm{S}$ e onda $\mathrm{T}$ e em $\mathrm{V} 2$ entre as ondas $\mathrm{R} \mathrm{e} \mathrm{S}$ de todos os grupos testados. 
Em V3 foi verificada diferença na onda $\mathrm{R}$ dos grupos $\mathrm{TZ}$ e CF, no entanto, tanto a onda R do grupo TZ quanto a do grupo CF não diferenciaram significativamente dos outros grupos testados (ISO e CET). Em relação a onda S não foi observada diferença estatisticamente significativa.

A derivação V4 não apresentou diferença entre o complexo QRS e onda T entre todos os grupos.

A distribuição das alterações de acordo com o protocolo de contenção realizado está disposta no Quadro 3. 0 grupo TZ apresentou 3 alterações (7,5\%), em ISO foram encontradas 4 alterações (10\%), em CET 6 alterações (15\%) enquanto que no grupo $\mathrm{CF}$ foram constatadas 7 alterações $(17,5 \%)$. Não foi observada diferença estatisticamente significativa entre as frequências de ocorrências de alterações nos protocolos utilizados (Qui-quadrado, $\mathrm{p}=0,221$ ).

\section{DISCUSSÃO}

Os valores da frequência cardíaca (FC) observados neste trabalho estão de acordo com os encontrados por Baer (1994), Rajendra et al. (2010) e Galante (2013) em Aotus submetidos à anestesia, no entanto foram considerados elevados em comparação aos dados obtidos por Smith \& Astley (2007) em Aotus nancymai em condição de repouso. Sabe-se que, tanto os anestésicos utilizados quanto fatores estressantes como a contenção física elevam a FC de forma considerável (Smith \& Astley 2007).

Dentre os anestésicos utilizados a cetamina é o que mais eleva a FC (Ozturk et al. 1999). Apesar de a tiletamina pertencer ao mesmo grupo farmacológico da cetamina, a associação tiletamina/zolazepam produz efeitos cardiovasculares menores, enquanto que o isoflurano pode provocar uma ligeira redução na FC (Natalini 2007), porém neste trabalho este parâmetro não variou entre os grupos testados.

Quadro 1. Média e desvio padrão dos parâmetros eletrocardiográficos de Aotus azarae infulatus submetidos à contenção com tiletamina/zolazepam (TZ), isoflurano (ISO), cetamina e midazolam (CET) e contenção física (CF). Parâmetros gravados na derivação DII em $50 \mathrm{~mm} / \mathrm{s}$ e N = 1 $(1 \mathrm{~cm}=1 \mathrm{mV})$

\begin{tabular}{|c|c|c|c|c|}
\hline \multirow[t]{2}{*}{ Parâmetros } & \multicolumn{4}{|c|}{ Grupo } \\
\hline & $\mathrm{TZ}$ & ISO & CET & $\mathrm{CF}$ \\
\hline $\mathrm{FC}(\mathrm{bpm})$ & $262 \pm 43,66$ & $250 \pm 28,67$ & $284 \pm 24,58$ & $258 \pm 67,62$ \\
\hline \multicolumn{5}{|c|}{ 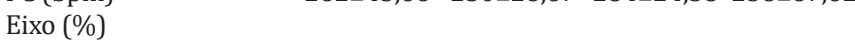 } \\
\hline Normal $\left(60-90^{\circ}\right)$ & $70 \%$ & $70 \%$ & $50 \%$ & $80 \%$ \\
\hline Alterado & $30 \%$ & $30 \%$ & $50 \%$ & $20 \%$ \\
\hline \multicolumn{5}{|l|}{ Onda P } \\
\hline Duração (s) & $0,04 \pm 0,01$ & $0,04 \pm 0,01$ & $0,05 \pm 0,01$ & $0,04 \pm 0,01$ \\
\hline Amplitude (mV) & $0,24 \pm 0,06$ & $0,19 \pm 0,06$ & $0,20 \pm 0,10$ & $0,15 \pm 0,05$ \\
\hline Intervalo PR (s) & $0,08 \pm 0,01$ & $0,07 \pm 0,01$ & $0,08 \pm 0,01$ & $0,08 \pm 0,01$ \\
\hline \multicolumn{5}{|l|}{ Complexo QRS } \\
\hline Duração (s) & $0,05 \pm 0,01$ & $0,04 \pm 0,01$ & $0,05 \pm 0,01$ & $0,04 \pm 0,01$ \\
\hline Amplitude (mV) & $0,75 \pm 0,41$ & $0,71 \pm 0,32$ & $0,78 \pm 0,33$ & $0,74 \pm 0,37$ \\
\hline Segmento S-T* & $0,05 \pm 0,10$ & $0,09 \pm 0,16$ & $0,10 \pm 0,08$ & $0,09 \pm 0,08$ \\
\hline Intervalo QT (s) & $0,14 \pm 0,03$ & $0,15 \pm 0,01$ & $0,13 \pm 0,01$ & $0,14 \pm 0,03$ \\
\hline \multicolumn{5}{|c|}{ Polaridade da onda T (\%) } \\
\hline Positiva & $90 \%$ & $90 \%$ & $90 \%$ & $100 \%$ \\
\hline Negativa & 0 & $10 \%$ & $10 \%$ & 0 \\
\hline Isoelétrica & $10 \%$ & 0 & 0 & 0 \\
\hline
\end{tabular}

* No segmento S-T valores em infradesnível foram considerados negativos, enquanto que valores em supradesnível foram considerados positivos, relacionados à linha de base do ECG.
Quadro 2. Dados obtidos a partir das precordiais do ECG em Aotus azarae infulatus submetidos à contenção com tiletamina/zolazepam (TZ), isoflurano (ISO), cetamina e midazolam (CET) e contenção física (CF). Parâmetros gravados em $50 \mathrm{~mm} / \mathrm{s}$ e $\mathrm{N}=1(1 \mathrm{~cm}=1 \mathrm{mV})$

\begin{tabular}{lcccc}
\hline Precordiais & \multicolumn{4}{c}{ Grupos } \\
\cline { 2 - 5 } V1 & TZ & ISO & CET & CF \\
\hline Onda R & $0.37 \pm 0.12$ & $0.37 \pm 0.20$ & $0.50 \pm 0.37$ & $0.22 \pm 0.10$ \\
Onda S* & $0.02 \pm 0.08$ & $0.03 \pm 0.20$ & $0.10 \pm 0.23$ & $0.01 \pm 0.14$ \\
Onda T** & & & & \\
Positiva & $80 \%$ & $60 \%$ & $50 \%$ & $50 \%$ \\
Negativa & $10 \%$ & $40 \%$ & $40 \%$ & $20 \%$ \\
V2 & & & & \\
Onda R & $0.97 \pm 0.43$ & $0.74 \pm 0.33$ & $1.02 \pm 0.54$ & $1.05 \pm 0.44$ \\
Onda S* & $0.13 \pm 0.16$ & $0.08 \pm 0.02$ & $0.16 \pm 0.06$ & $0.08 \pm 0.02$ \\
V3 & & & & \\
Onda R & $0.77 \pm 0.24$ & $0.51 \pm 0.34$ & $0.5 \pm 0.29$ & $0.41 \pm 0.18$ \\
Onda S* & $0.28 \pm 0.24$ & $0.06 \pm 0.09$ & $0.14 \pm 0.00$ & $0.15 \pm 0.07$ \\
V4 & & & &
\end{tabular}

V4

Complexo QRS (\%)

Positiva

Negativa

Onda T (\%)

Positiva

Negativa

$\begin{array}{cccc}90 \% & 90 \% & 60 \% & 90 \% \\ 10 \% & 10 \% & 40 \% & 10 \% \\ & & & \\ 00 \% & 60 \% & 80 \% & 90 \% \\ 0 & 40 \% & 20 \% & 10 \%\end{array}$

* Na onda S os valores em infradesnível foram considerados negativos, enquanto que valores em supradesnível foram considerados positivos, relacionados à linha de base do ECG.

** Onda $\mathrm{T}=10 \%$ não visível em $\mathrm{TZ}, 10 \%$ não visível em CET, 30\% não visível em $\mathrm{CF}$.

Quadro 3. Relação entre as alterações encontradas no

ECG em Aotus azarae infulatus de acordo com o tipo de contenção realizada: tiletamina/zolazepam (TZ), isoflurano (ISO), cetamina e midazolam (CET) e contenção física (CF)

\begin{tabular}{|c|c|c|c|c|}
\hline \multirow[t]{2}{*}{ Alteração } & \multicolumn{4}{|c|}{ Grupos } \\
\hline & $\mathrm{TZ}$ & ISO & CET & $\mathrm{CF}$ \\
\hline Onda $\mathrm{T} \geq \mathrm{QRS}$ & 1 & 2 & 2 & 5 \\
\hline Batimento de Fusão (P-T) & 1 & & & \\
\hline P negativo com ritmo juncional & 1 & 1 & 1 & \\
\hline Segmento S-T elevado & & 1 & & \\
\hline Segmento S-T abobadado & & & 1 & \\
\hline Contração atrial prematura & & & 2 & \\
\hline Arritmia sinusal com marca-passo migratório & & & & 1 \\
\hline Ausência de onda P & & & & 1 \\
\hline * Total de ECG por grupo & 10 & 10 & 10 & 10 \\
\hline ** Percentual de alterações & $7,5 \%$ & $10 \%$ & $15 \%$ & $17,5 \%$ \\
\hline
\end{tabular}

A anestesia pode ser uma fonte de variabilidade nos resultados do ECG, no entanto os parâmetros obtidos do traçado eletrocardiográfico de todos os grupos testados estão de acordo com os dados citados por Baer (1994) e Rajendra et al. (2010) em Aotus, sob contenção química, não sendo encontrados até presente momento, trabalhos que forneçam dados de ECG em condições de vida livre na espécie Aotus azarae infulatus.

Não existe consenso em relação ao posicionamento de primatas para a realização do ECG, sendo adotada posição supina do animal, de forma semelhante ao descrito por Yang et al. (2011). A duração da onda P verificada corrobora com o descrito por Baer (1994) em Aotus mas foi maior 
do que o citado por Rajendra et al. (2010) nesta mesma espécie. $O$ intervalo $\mathrm{P}-\mathrm{R}$, o complexo QRS e o intervalo Q-T encontrados estão de acordo aos relatados por Baer (1994) e Rajendra et al. (2010).

Não foram encontrados valores de referência de derivações precordiais em primatas, o que impossibilitou a discussão desses valores. Desta forma, esta pesquisa limitou-se a apresentar os resultados obtidos com as derivações precordiais para que sirvam como informação para pesquisas posteriores.

Segundo Booth (1992), a cetamina não induz maiores alterações no padrão do ECG de primatas não-humanos, no entanto foram observadas seis alterações no grupo CET. Apesar de o isoflurano ser um anestésico indicado para pacientes com alterações cardíacas, o Grupo ISO também promoveu alterações, o que está de acordo com o citado por Joyner et al. (2008) em águias. Isso pode ter ocorrido devido ao estresse causado pela indução com máscara e pelo odor pungente do agente, fato este também observado por Moutinho (2010) em coelhos.

Apesar de não ter havido diferença estatística entre as alterações observadas, houve uma tendência do Grupo TZ a apresentar melhores resultados, apresentando menor número de alterações no traçado eletrocardiográfico em relação aos demais. Acredita-se que este resultado ocorreu devido à quantidade de animais empregados nesta pesquisa, sendo necessários estudos com um número maior de animais para esclarecer este fato.

Não foram encontrados relatos de alterações com o uso da associação tiletamina/zolazepam ou com a contenção física no ECG de primatas, porém neste estudo foi observado que tanto o Grupo TZ quanto o Grupo CF, apresentaram alterações, sendo este último o que mais promoveu alterações. Isso provavelmente ocorreu devido ao grande estresse a que os animais foram submetidos durante a contenção física.

\section{CONCLUSÕES}

0 presente estudo realizado com macacos-da-noite ( $\mathrm{Ao}$ tus azarae infulatus) permitiu concluir que os protocolos de contenção empregados não alteraram os valores do ECG.

Observou-se que o grupo contenção física não pôde ser considerado como controle, visto que promoveu mais alterações do que os outros protocolos de contenção testados.

\section{REFERÊNCIAS}

Baer J.F. 1994. Husbandry and medical management of the owl monkey, p.133-164. In: Baer J.F., Weller R.E. \& Kakaoma I. (Eds), Aotus: the owl monkey. Academic Press, San Diego.
Booth N.H. 1992. Anestésicos intravenosos e outros parenterais, p.168218. In: Booth N.H. \& McDonald L.E. (Eds), Farmacologia e Terapêutica em Veterinária. 6aㅡ ed. Guanabara Koogan, Rio de Janeiro. 997p.

Chaves P.C. \& Moreira A.L. 2001. Electrocardiografia. Aula Teórico-Prática, Faculdade de Medicina da Universidade do Porto, Portugal. 26p.

Del-Claro K. \& Prezoto F. 2003. As distintas fases do comportamento animal. Sociedade Brasileira de Etologia, Conceito, Jundiaí, SP. 276p.

Galante R. 2013.Anestesia intravenosa total em primatas: comparação da infusão contínua de propofol com bolus intravenosos de tiletamina e zolazepam e associação de propofol com opioides ou cetamina. Dissertação de Mestrado em Medicina Veterinária, Universidade Federal do Paraná, Curitiba. 218p.

ICMBIO 2011. Sumário executivo do plano de ação nacional para a conservação dos primatas do Nordeste. Instituto Chico Mendes. Disponível em $<$ http://www.icmbio.gov.br> Acesso em fev. 2013.

Joyner P.H., Jones M.P., Ward M., Gompf R.E., Zagaya N. \& Sleeman J.M. 2008. Induction and recovery characteristics and cardiopulmonary effects of sevoflurane and isoflurane in bald eagles. Am. J. Vet. Res. 69(1): 13-22.

Menezes A.N., Bonvicino C.R. \& Seuanez H.N. 2010. Identification, classification and evolution of owl monkeys (Aotus Illiger, 1811). BMC Evolutionary Biology 10:248.

Monteiro F.O.B. 2004. Avaliação uterina e diagnóstico de gestação em macaco-da-noite (Aotus azarae infulatus) por ultra-som em modo B. Dissertação de Mestrado em Medicina Veterinária, Faculdade de Ciências Agrárias e Veterinárias, Unesp, Jaboticabal. 70p.

Monteiro F.O.B., Koivisto M.B., Vicente W.R., Amorim C.R., Whiteman C.W., Castro P.H. \& Maia C. E. 2006. Uterine evaluation and gestation diagnosis in owl monkey (Aotus azarai infulatus) using the B mode ultrasound. J. Med. Primatol. 35(3):123-130.

Moutinho I.I.M. 2010. Estudo comparativo dos anestésicos sevoflurano vs isoflurano em coelhos (Oryctolagus cuniculi). Dissertação de Mestrado em Medicina Veterinária, Faculdade de Medicina Veterinária, Universidade Técnica de Lisboa, Portugal. 123p.

Natalini C.C. 2007. Teorias e técnicas em anestesiologia veterinária, p.5356. ArtMed, Porto Alegre. 296p.

Ozturk T., Tuncok Y., Kalkan S., Guven H. \& Aran G. 1999. Midazolams cardiac depressant effects andtheir lack of reversal by flumazenil in isolated rabbit hearts. Pharmacol. Res. 39(4):283-287.

Rajendra R.S., Brady A.G., Parks V.L., Massey C.V., Gibson S.V. \& Abee C.R. 2010. The normal and abnormal owl monkey (Aotus sp.) heart: looking at cardiomyopathy changes with echocardiography and electrocardiography. J. Med. Primatol. 39(3):143-150.

Silva A.L.P. \& Ferreira H.I. 1994. 0 uso do cloridrato de detomidina em cães. Cães Gatos 9:18-20.

Smith O.A. \& Astley C.A. 2007. Naturally occurring hypertension in New World nonhuman primates: potential role of the perifornical hypothalamus. Am. J. Physiol., Regulatory Integrative and Comp. Physiol. 292(2):937-945.

Yang P., Han P., Hou J., Zhang L., Song H., Xie Y., Chen Y., Xie H., Gao F. \& Kang Y.J. 2011. Electrocardiographic characterization of rhesus monkey model of ischemic myocardial infarction induced by left anterior descending artery ligation. Cardiovasc. Toxicol. 11(4):365-372. 